\title{
Evaluación del olfato en pacientes con patología tumoral en la región selar, intervenidos mediante abordaje endoscópico transesfenoidal transnasal
}

\section{Assessment of smell in patients with tumor pathology in the sellar region, operated by transnasal transsphenoidal endoscopic approach}

\author{
Nadia Karin Vega A', Katherine Walker J', J. Paul Boettiger B'
}

\begin{abstract}
RESUMEN
Introducción: Para acceder a la región selar, podemos utilizar las técnicas transcraneal, transeptal, o transnasal endoscópica, pudiendo provocar diferentes grados de hiposmia. Se ha descrito menor morbilidad al utilizar la técnica endoscópica, pero faltan estudios dirigidos a los resultados olfatorios.

Objetivo: Determinar la presencia de deterioro olfatorio en los pacientes sometidos a un abordaje transnasal endoscópico.

Material y método: Se reclutaron 12 pacientes con tumores en la región selar durante 8 meses. Se les realizó un test de olfato preoperatorio, fueron intervenidos mediante abordaje transnasal endoscópico y controlados al mes posoperatorio.

Resultados: Se logró seguimiento a 10 pacientes. Seis (60\%) presentaron un test de olfato preoperatorio normal. Al mes posoperatorio, se constató mejoría olfatoria en $1(10 \%)$ paciente, 8 (80\%) se mantuvieron en la misma categoría y 1 (10\%) presentó deterioro olfatorio. En suma, 9 de 10 pacientes (90\%) mantienen o mejoran su olfato al mes posoperatorio.

Conclusión: Nuestros resultados sugieren que el abordaje transnasal endoscópico utilizado en este estudio no produce deterioro olfatorio. Dado que además es una técnica de abordaje efectiva y relativamente segura, consideramos que constituye una alternativa factible para utilizar en pacientes con patología tumoral en la región selar.
\end{abstract}

Palabras claves: Olfato, hiposmia, abordaje endoscópico transnasal transesfenoidal, Sniffin test, mucosa olfatoria, región selar.

\section{ABSTRACT}

Introduction: To access the sellar region we can use the transcranial, transeptal, or transnasal endoscopic approaches, which may cause different degrees of hyposmia. It has described less morbidity to use the endocopic technique, however, there are few studies directed at olfactory outcomes.

Aim: To determine the presence of olfactory impairment secondary to endoscopic transnasal approach.

${ }^{1}$ Médicos del Servicio de Otorrinolaringología Hospital Clínico Universidad de Chile. 
Material and method: 12 patients with tumors in the sellar region were enrolled during 8 months. They were underwent a smell test preoperatively, operated by endoscopic transnasal approach, and controlled with postoperative retesting, after one month.

Results: Follow-up was achieved to 10 patients. $6(60 \%)$ presented a normal preoperative smell test. Within one postoperative month, olfactory improvement was found in $1(10 \%)$ patient, 8 (80\%) remained in the same category and $1(10 \%)$ had olfactory impairment. In all, 9 out of 10 patients (90\%) maintain or improve their sense of smell after surgery.

Conclusions: Our results suggest that the transnasal endoscopic approach used in this study, doesn't produce olfactory impairment. Given that is also an effective and relatively safe approach, we believe that is a feasible alternative for use in patients with tumor pathology in the sellar region.

Key words: Smell, transnasal transsphenoidal endoscopic approach, Sniffin sticks Screening Test, olfactory mucosa.

\section{INTRODUCCIÓN}

El sentido del olfato incluye funciones que van más allá de la percepción de olores. Es conocida su participación en la percepción de sabores, su rol primario como mecanismo de protección contra eventos potencialmente peligrosos (detección de alimentos en descomposición, fugas de gas, entre otros) y su contribución al establecimiento de la memoria afectiva y las relaciones interpersonales. En consecuencia, el deterioro del olfato se asocia a una considerable disminución en la calidad de vida ${ }^{1}$.

Los procedimientos quirúrgicos realizados en la región selar pueden producir grados variables de hiposmia. Si bien las técnicas de abordaje han evolucionado hacia prácticas menos invasivas y simplificadas, no están exentas de generar alteraciones permanentes en el olfato.

En sus inicios, el abordaje se realizaba exclusivamente vía transcraneal2 ${ }^{2}$. Actualmente, esta técnica se utiliza para casos seleccionados, como tumores invasores con extensión intracavernosa ${ }^{3}$ o tumores de gran tamaño cuya resección por vía transnasal ha sido incompleta. Desde el año 1910, se comienza a utilizar el abordaje transesfenoidal transeptal mostrando resultados quirúrgicos comparables al abordaje transcraneal, pero con una menor tasa de morbilidad y mortalidad posoperatoria². En el año 1907, Hermann Schloffer realiza el primer abordaje transesfenoidal transnasal y desde entonces, se han incorporado numerosos avances que han perfeccionado la técnica, como el uso de endoscopio nasal por Jankowski en $1992^{2,4}$.
La técnica transnasal endoscópica consiste en acceder directamente a través de las fosas nasales, resecar un segmento óseo del septum y de la mucosa nasal adyacente para acceder al esfenoides y, a través de éste, a la región selar ${ }^{5}$. La resección septal posterior varía de acuerdo a la técnica utilizada pudiendo ser desde una resección de mucosa unilateral 0 bilateral de unos pocos centímetros hasta la realización de un gran colgajo septal como el colgajo de Hadad, que permite cubrir amplias zonas de la región selar especialmente en los casos de alto riesgo de fístula de líquido cefalorraquíde $0^{6}$. Se ha descrito que la morbilidad asociada es menor en relación a otras técnicas, principalmente por prescindir de incisiones externas y sublabiales. Además, dado que en la mayoría de los casos la disección de mucosa es restringida y la mayoría de las veces unilateral, tendría similar y escaso impacto sobre el olfato al comparar con la técnica transesfenoidal transeptal ${ }^{4}$. Aun así, es razonable pensar que al realizar un abordaje endoscópico y resecar tejidos en un área donde sabemos que se localiza mucosa olfatoria, podríamos lesionar esta misma y con ello, deteriorar el olfato.

Considerando que existen pocos estudios dirigidos a los resultados olfatorios de las diferentes técnicas, se necesitan nuevas investigaciones que permitan establecer con mayor claridad el impacto real de estas intervenciones sobre el olfato.

\section{OBJETIVO}

El objetivo de este estudio es determinar la presencia de deterioro olfatorio posoperatorio en 
Ios pacientes intervenidos mediante el abordaje endoscópico transesfenoidal transnasal.

\section{MATERIAL Y MÉTODO}

Estudio de una cohorte de pacientes con patología tumoral en la región selar, intervenidos por un equipo combinado de otorrinolaringólogo y neurocirujano mediante un abordaje endoscópico transesfenoidal transnasal, a quienes se les realizó la evaluación del olfato utilizando un test estandarizado (Sniffin Sticks Screening Test ${ }^{\circledR}$. Este estudio fue realizado en el Hospital Clínico Universidad de Chile.

Se reclutaron 12 pacientes candidatos a cirugía por patología tumoral en la región selar desde junio de 2010 hasta febrero de 2011, a quienes se realizó un test de olfato previo a la intervención, y luego un nuevo test al mes posoperatorio. Todos los pacientes accedieron a participar del estudio en forma voluntaria, por lo que se obtuvo su autorización a través del consentimiento informado correspondiente.

Respecto a los antecedentes médicos, destacaron 4 pacientes con cirugía selar previa y 4 pacientes con patología rinosinusal (concha bulosa, desviación septal, poliposis nasosinusal e hipertrofia de cornetes).

El instrumento utilizado para evaluar el olfato (Sniffin Sticks Screening Test ${ }^{\circledR}$, consiste en 12 dispositivos tipo lápiz con dispensador de olor, que son presentados a los pacientes por 3 segundos a una distancia de $2 \mathrm{cms}$ de las fosas nasales. El paciente debe identificar el olor entre 4 alternativas que se le presentan. Debe considerarse un intervalo mínimo de 30 segundos entre cada exposición para evitar la desensibilización olfatoria ${ }^{7}$.

En relación al procedimiento, fue realizado por el equipo de Otorrinolaringología del Hospital Clínico Universidad de Chile. La cirugía fue estandarizada en todos los pacientes. Se realizó infiltración con solución de adrenalina al 1/100.000, luego se procedió a la lateralización de los cornetes inferior y medio, con lateralización o resección del cornete superior. Posteriormente, se realizó la búsqueda del ostium esfenoidal en forma visual o por palpación mediante tactador, procediendo a la cauterización de la mucosa adyacente y ampliación del ostium con Kerrison. Luego, se realiza la resección del septum óseo anterior al rostrum y de la mucosa septal adyacente ipsilateral. Se continúa con la resección del rostrum con fresa 0 cincel para ampliar la apertura de la cara anterior del esfenoides y permitir una buena exposición de la silla turca, los relieves carotídeos y los nervios ópticos. El equipo neuroquirúrgico realiza la resección del tumor. Finalmente, se realiza la hemostasia correspondiente, utilizando aspirador cauterio y Surgiflo ${ }^{\circledR}$ sobre la mucosa cruenta y se procede a la medialización de los cornetes.

Respecto a los resultados, se catalogó al paciente según la edad y el puntaje alcanzado en el test como olfato normal, hiposmia o anosmia según el estudio de validación realizado por Hudson y cols ${ }^{7}$ en población chilena, donde se considera olfato normal un puntaje de 9 a 12 en población entre 21 y 39 años, 8 a 12 puntos entre 40 y 59 años y 7 a 12 puntos en personas de 60 años y más. Se considera anosmia un puntaje menor 0 igual a 6 en población entre 21 y 39 años, menor 0 igual a 5 entre 40 y 59 años, y menor 0 igual a 4 en personas de 60 años y más. Los valores intermedios califican en el rango de hiposmia.

Se consideró mejoría olfatoria si el paciente pasaba de categoría hiposmia a normal según el resultado del test posoperatorio. Se consideró deterioro olfatorio si el paciente pasaba de categoría normal a hiposmia o anosmia en el posoperatorio. Cambios en el puntaje del test que no involucraron un cambio de categoría olfatoria, no se consideraron como mejoría ni deterioro. Los datos se presentan en medianas como medida de resumen. Se utilizó el test Wilcoxon Rank-Sum para determinar diferencias en el resultado del test de olfato pre y posoperatorio. Se utilizó una significancia de 0,05 y los datos fueron procesados mediante el programa Stata ${ }^{\circledR}$ V.11.1

\section{RESULTADOS}

De los 12 pacientes reclutados al inicio del estudio, dos fueron excluidos (por anosmia preoperatoria y abandono de seguimiento). La edad promedio al momento de la cirugía fue de 37,5 años (rango de 21 a 64 años). La muestra de pacientes con seguimiento al mes del posoperatorio fue de 10 pacientes, $6(60 \%)$ de sexo femenino y 4 $(40 \%)$ de sexo masculino. 
En relación a la técnica quirúrgica, se realizó resección hemitransfixiante de mucosa septal y septum óseo posterior de $\pm 2 \mathrm{cms}$. en toda la muestra (10 pacientes). Se realizó resección de cornete superior en 1 paciente y resección de cornete medio en 2 pacientes. Ningún paciente requirió la realización de colgajo septal (Hadad).

Respecto al diagnóstico anatomopatológico, 3 (30\%) pacientes fueron informados como adenoma hipofisiario no funcionante, $6(60 \%)$ pacientes como adenoma hipofisiario funcionante y $1(10 \%)$ paciente fue informado como cordoma. En la Tabla 1 y Figura 1 se muestran los diagnósticos anatomopatológicos.

El análisis del olfato preoperatorio, mostró que $6(60 \%)$ pacientes se encontraban en rango normal y 4 (40\%) pacientes en rango de hiposmia. Todos los pacientes con hiposmia presentaban patología rinosinusal en forma concomitante (poliposis nasosinusal; hipertrofia de cornetes; concha bulosa más desviación septal; concha bulosa bilateral, respectivamente). Al mes posoperatorio, se constató nuevamente $6(60 \%)$ pacientes en rango normal y 4 (40\%) pacientes en rango de hiposmia.
Las medianas del test de olfato preoperatorio (9 puntos) y posoperatoria ( 9,5 puntos) no muestran diferencias significativas $(p=0,13)$.

Respecto a la muestra, destacan dos pacientes. Uno de ellos mostró mejoría olfatoria en el test posoperatorio (de 8 a 11 puntos) pasando de categoría hiposmia a normal. Es importante mencionar que este paciente presentaba poliposis nasosinusal en el preoperatorio y que fue intervenido mediante una cirugía endoscópica funcional (CEF) previo al abordaje transesfenoidal, en el mismo tiempo operatorio.

Otro paciente, mostró deterioro olfatorio (de 9 a 7 puntos) pasando de rango normal a hiposmia. En el protocolo operatorio, se describe la realización de resección hemitransfixiante de septum, sin otras resecciones, y evolucionó sin otras complicaciones posoperatorias.

Los 8 pacientes restantes se mantuvieron en la misma categoría que indicó el test preoperatorio ( 5 pacientes en rango normal, 3 pacientes en rango de hiposmia). En suma, 9 de 10 pacientes mejoraron o mantuvieron su olfato en el periodo posoperatorio. En la Tabla 1 y Figura 2 se muestran los resultados.

Tabla 1. Características demográficas, anatomía patológica y resultados del test de olfato (Sniffin Sticks Screening Test ${ }^{\circledR}$ )

\begin{tabular}{|c|c|c|c|c|c|c|}
\hline Paciente & Edad & $\begin{array}{l}\text { Diagnóstico } \\
\text { anatomopatológico }\end{array}$ & Puntaje & Rango & Puntaje & Rango \\
\hline 1 & 64 & $\begin{array}{l}\text { Adenoma hipófisis } \\
\text { no funcionante }\end{array}$ & 11 & Normal & 12 & Normal \\
\hline 2 & 22 & Prolactinoma & 9 & Normal & 10 & Normal \\
\hline 3 & 21 & $\begin{array}{l}\text { Adenoma hipófisis } \\
\text { no funcionante }\end{array}$ & 12 & Normal & 12 & Normal \\
\hline 4 & 25 & Cordoma & 8 & Hiposmia & 11 & Normal \\
\hline 5 & 36 & $\begin{array}{l}\text { Adenoma hipófisis } \\
\text { funcionante (FSH) }\end{array}$ & 6 & Hiposmia & 8 & \\
\hline 6 & 52 & $\begin{array}{l}\text { Adenoma hipofisis } \\
\text { funcionante }(\mathrm{GH})\end{array}$ & 9 & Normal & 9 & Normal \\
\hline 7 & 29 & $\begin{array}{l}\text { Adenoma hipófisis } \\
\text { funcionante (ACTH) }\end{array}$ & 7 & Hiposmia & 8 & \\
\hline 8 & 54 & $\begin{array}{l}\text { Adenoma hipófisis } \\
\text { no funcionante }\end{array}$ & 9 & Normal & 7 & \\
\hline 9 & 29 & $\begin{array}{l}\text { Adenoma hipófisis } \\
\text { funcionante (ACTH) }\end{array}$ & 8 & Hiposmia & 8 & \\
\hline 10 & 43 & $\begin{array}{l}\text { Adenoma hipófisis } \\
\text { funcionante (FSH) }\end{array}$ & 10 & Normal & 10 & Normal \\
\hline
\end{tabular}




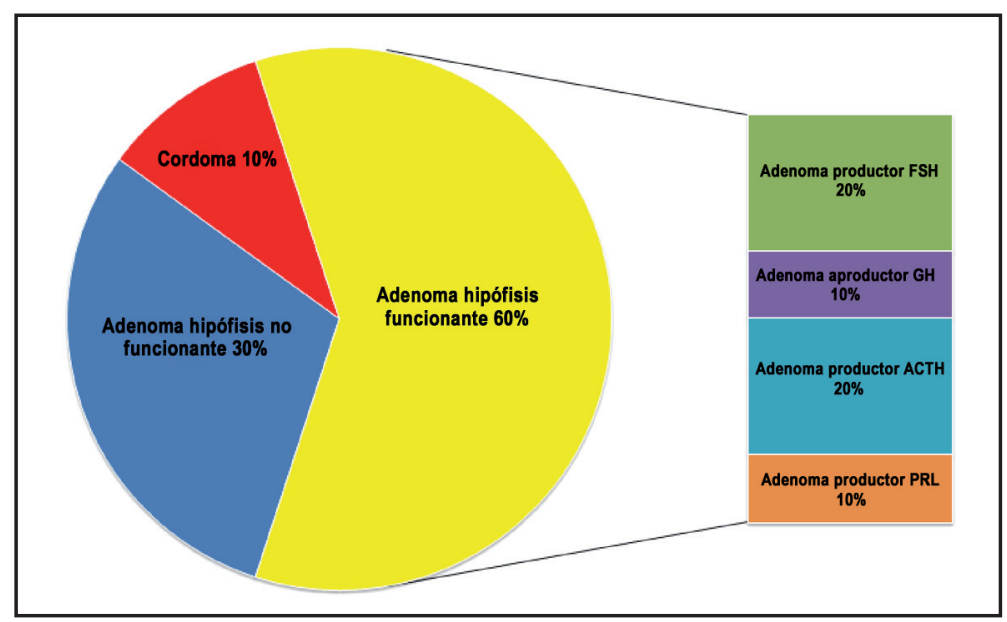

Figura 1. Gráfico diagnóstico anatomopatológico.

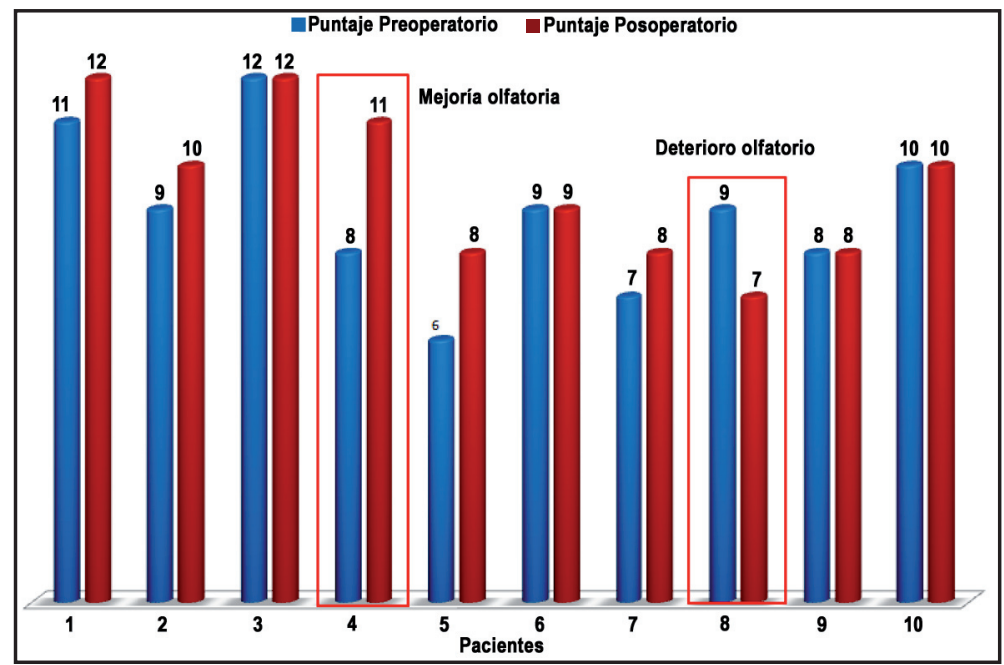

Figura 2. Resultados test olfato pre y posoperatorio.

En relación a la técnica quirúrgica, el paciente en que se realizó resección de cornete superior se mantuvo en la misma categoría que indicó el test preoperatorio (hiposmia). Los pacientes en que se realizó resección parcial de cornete medio, tampoco mostraron variación en su categoría según el test pre y posoperatorio.

En el seguimiento, las complicaciones pesquisadas fueron $4(40 \%)$ pacientes con sinequias posoperatorias y $2(20 \%)$ pacientes con epistaxis leve.

\section{DISCUSIÓN}

A pesar de que existen múltiples técnicas para acceder a la región selar, los avances en el ámbito quirúrgico están orientados a seleccionar las técnicas menos invasivas y con menor porcentaje de complicaciones para realizar este tipo de intervenciones. En este estudio, se ha propuesto el abordaje endoscópico transesfenoidal transnasal como una alternativa factible y segura. Sin embargo, incluye resecciones en la región nasal que 
pudieran comprometer la mucosa olfatoria y con ello deteriorar el olfato.

Clásicamente, se ha descrito que la mucosa olfatoria se extiende en la porción póstero-superior de la bóveda nasal, cubriendo la porción superior del septum, lámina cribiforme, cornete superior y algunas áreas del cornete medio ${ }^{8}$. Sin embargo, estudios recientes en mucosa olfatoria, han mostrado que su localización y extensión precisa puede variar entre los individuos y a través del tiempo8 ${ }^{8-9}$, por lo que resulta difícil establecer una relación directa a priori entre el tejido lesionado durante el procedimiento y el porcentaje de mucosa olfatoria afectada.

Según nuestros resultados, no existen diferencias significativas entre el olfato pre y posoperatorio evaluado con el test de olfato Sniffin Sticks Screening Test ${ }^{\circledR}$. Esto podría deberse a que la resección de mucosa nasal es restringida con el objetivo de minimizar el daño, y que en todos los pacientes se realizó una resección hemitransfixiante conservadora de mucosa septal, preservando la mucosa olfatoria del lado contralateral. Sin embargo, no podemos dejar de mencionar las limitaciones dadas por el pequeño tamaño de la muestra al extrapolar los resultados.

Los estudios de Kennedy y cols ${ }^{10}$ e Ikeda y cols ${ }^{11}$ realizados con técnica transesfenoidal transeptal, reportaron deterioro olfatorio hasta en el $12 \%$ de sus pacientes. Es importante señalar que en estos estudios, el deterioro olfatorio se determinó mediante el reporte de pérdida olfatoria por parte de los mismos pacientes, sin utilizar un test de olfato estandarizado.

Respecto a los resultados olfatorios de la técnica transesfenoidal transnasal endoscópica, destacan dos estudios realizados en población norteamericana. Uno de ellos, Rotenberg y cols ${ }^{12}$, realizaron un estudio publicado en el año 2011, con 17 pacientes, quienes fueron evaluados con el test de olfato de la Universidad de Pennsylvania (UPSIT) en el periodo preoperatorio y a los 6 meses posoperatorio. En esta serie, se realizó colgajo septal de Hadad en todos los pacientes, encontrando en todos ellos un deterioro del olfato calificado como hiposmia moderada a los 6 meses. Hart y cols ${ }^{4}$, realizaron un estudio publicado en el año 2010, con 57 pacientes a quienes realizaron el test de olfato UPSIT preoperatorio, al mes y tres meses posabordaje endoscópico, observando que el $64 \%$ de los pacientes presentaba una disminución transitoria del olfato durante el primer mes. En la evaluación al tercer mes, no se constataron diferencias significativas entre los puntajes preoperatorio y posoperatorio, atribuyendo este deterioro olfatorio transitorio al edema y procesos de cicatrización posoperatoria. En este estudio se realizó resección posterior de septum pero no se menciona la realización de colgajo septal de Hadad en ninguno de los pacientes.

El colgajo pediculado neurovascular de mucoperiostio y mucopericondrio septal (Hadad), se realiza para disminuir la incidencia de fístula de líquido cefalorraquídeo (LCR) posoperatoria. En general, se recomienda en la reconstrucción de grandes defectos durales de base de cráneo y en pacientes en que se planea realizar radioterapia posoperatoria ${ }^{6}$. Al realizar este colgajo, frecuentemente se incorpora tejido de la porción superior del septum, que contiene mucosa olfatoria ${ }^{12}$. Esto explicaría porque en la serie de casos de Rotenberg todos los pacientes experimentaron un deterioro del olfato, mientras que en nuestra serie y en la de Hart y cols, donde no se realizó este colgajo, los pacientes mantuvieron su nivel de olfato en el posoperatorio.

En relación a la seguridad de este abordaje, podemos plantear que constituye una alternativa relativamente segura para los pacientes, ya que en nuestra experiencia solo se evidenciaron complicaciones menores. Existen reportes en la literatura que comparan el abordaje transnasal endoscópico respecto al abordaje transeptal, mostrando una menor tasa de morbilidad posoperatoria (fístula de LCR, diabetes insípida, uso de drenaje lumbar y dolor $)^{13-14}$, menor tiempo operatorio ${ }^{5}$ y menor estadía hospitalaria en el grupo sometido al abordaje transnasal endoscópico ${ }^{13-14}$.

Por último, en relación al test de olfato, mencionar la importancia de la estandarización de los resultados según el grupo etario. Estudios señalan que tanto el número de receptores olfatorios como la altura del neuroepitelio disminuyen con la edad, asociándose a una disminución fisiológica de la sensibilidad olfatoria ${ }^{7-9}$, por lo tanto, la edad constituye una importante variable a considerar en la interpretación de los resultados del test de olfato.

El Sniffin Sticks Test ${ }^{\circledR}$ desarrollado por Hummel con 20 dispositivos de aroma, y su variante Sniffin Sticks Screening Test ${ }^{\circledR}$ desarrollado por el mismo autor, con 12 dispositivos de aromas, ha mostrado 
validez en la medición de la función olfatoria en estudios realizados en población adulta tanto a nivel global como local ${ }^{7,15-16}$, y en nuestra experiencia, resultó ser una herramienta de fácil y relativamente rápida aplicación, por lo que constituye un buen elemento para introducir a la práctica habitual del especialista.

\section{CONCLUSIONES}

El análisis de los resultados del test de olfato pre y posoperatorio, sugieren que el abordaje transnasal endoscópico utilizado en nuestro estudio no produce deterioro olfatorio. Destacamos que en ninguno de nuestros pacientes se realizó colgajo septal de Hadad, lo que influiría negativamente en la preservación del olfato posoperatorio. Este hecho apoya la racionalidad en la decisión de realizar o no este colgajo, considerando que el deterioro olfatorio se asocia a un importante compromiso de la calidad de vida. Por lo tanto, concluimos que el abordaje endoscópico transnasal es una técnica efectiva, relativamente segura y que no produce deterioro olfatorio, por lo que constituye una alternativa factible para utilizar en pacientes con patología tumoral en la región selar.

\section{BIBLIOGRAFÍA}

1. Sobol S, Frenkiel S, Mouadeb D. Olfactory disfunction: What's that smell? Canadian Journal of Diagnosis 2002; 19: 55-63.

2. LiU JK, Kaushik D, Weiss MH, et al. The history and evolution of transsphenoidal surgery. $J$ Neurosurg 2011; 95: 1083-96.

3. Spallone A, González-González J.L, Mostes de Oca F, Verdial-VIDAL R. Adenomas hipofisarios con invasión intracavernosa. Resultados del abordaje transcraneal al seno cavernoso. Neurocirugía 2007; 18: 294-300.

4. Hart CK, Theodosopoulos PV, Zimmer LA. Olfactory changes after endoscopic pituitary tumor resection. Otolaryngol Head Neck Surg 2010; 142(1): 95-7.

5. Olavarría C, Stott C, Lemp M, Bustamante C,
Schmidt N, Emerich M. Comparación de dos técnicas quirúrgicas para abordaje de la región selar: Transeptal transesfenoidal versus transnasal directa. Rev Otorrinolaringol Cir Cabeza Cuello 2004; 64: 91-8.

6. Hadad G, Bassagasteguy L, Carrau R, et al. A Novel Reconstructive Technique After Endoscopic Expanded Endonasal Approaches: Vascular Pedicle Nasoseptal Flap. Laryngoscope 116: October 2006.

7. Hudson L, Silva Consuelo, Núñez JC, Gómez R, VEnEgas $P$. Evaluation of olfaction in healthy subjects using the sniffing sticks battery. Rev Méd Chile 2012; 140: 442-6.

8. Escada P, Lima C, Madeira da Silva J. The human olfactory mucosa. Eur Arch Otorhinolaryngol 2009; DOI 10.1007/s00405-009-1073-x.

9. Fahim Haider Jafari, Phil M. A histological study of human olfactory mucosa: Regional distribution and age related changes. Thesis University of Health Sciences, Lahore. 2006.

10. Kennedy DW, Cohn ES, Pape ID, et al. Transsphenoidal approach to the sella: the Johns Hopkins experience. Laryngoscope 1984; 94: 1066-74.

11. Ikeda K, Watanabe K, Suzuki H, et al. Nasal airway resistance and olfactory acuity following transsphenoidal pituitary surgery. Am J Rhinol 1999; 13: 45-8.

12. Rotenberg BW, Saunders $S$, Duggal N. Olfactory Outcomes After Endoscopic Transsphenoidal Pituitary Surgery. Laryngoscope 2011; 121: 1611-3.

13. Neal JG, Patel SJ, Kulbersh JS, et al. Comparison of techniques for transsphenoidal pituitary surgery. Am J Rhinol 2007; 21: 203-6.

14. Higgins tS, Courtemanche C, Karakla D et al. Analysis of transnasal endoscopic versus transseptal microscopic approach for excision of pituitary tumors. Am J Rhinol 2008; 22: 649-52.

15. Hummel T, Sekinberg B, Wolf SR. Sniffin stick: Olfactory performance by the combined testing of odor identification, odor discrimination and olfactory threshold. Chem Senses 1997; 22: 39-52.

16. Haehner A, Mayer A, Landis B y col. High Test-Retest Reliability of the Extended Version of the "Sniffin' Sticks" Test. Chem Senses 2009; 34: 705-11.

Dirección: Katherine Walker L.

Hospital Clínico Universidad de Chile

E mail: kwalkerj007@gmail.com 